environment. The relatively simple cell culture systems that Kousteni et al. (9, 12) have examined to date are unlikely to mimic the complex interactions that regulate the skeleton in vivo. It also is unclear whether the effects of sex steroid hormone withdrawal on the skeleton are mediated predominantly by the ability of these agents to regulate the differentiation of osteoblasts and osteoclasts from their precursor cells or, as Kousteni et al. postulate (12), the ability of sex steroid hormones to regulate apoptosis. There is now strong evidence in murine models that estrogen withdrawal is associated with an increased number of osteoclast precursor cells in the marrow (10), an effect that has been linked to the regulation of B-lymphopoiesis (13-15). Since the target cell for this response is unknown, it is unclear what pathways estren or estrogen uses to produce this response. Hence, studies of the mechanism by which sex steroid hormones or estren mediate their bone-sparing effects ultimately will require in vivo experiments that use sophisticated technologies to dissect in detail both the cell-specific and the subcellular compartment-specific effects that these agents have on bone mass. Once data from such in vivo studies become available, a more complete understanding of the many effects that sex steroid hormones have on the skeleton should be appreciated.

1. Cato, A.C., Nestl, A., and Mink, S. 2002. Rapid actions of steroid receptors in cellular signaling pathways. Sci. STKE. 138:RE9.

2.Shupnik, M.A. 2002. Oestrogen receptors, receptor variants and oestrogen actions in the hypothalamic-pituitary axis. J. Neuroendocrinol. 14:85-94.

3. Riggs, B.L., and Hartmann, L.C. 2003. Selective estrogen-receptor modulators: mechanisms of action and application to clinical practice. N. Engl. J. Med. 348:618-629.

4. Paech, K., et al. 1997. Differential ligand activation of estrogen receptors ERalpha and ERbeta at AP1 sites. Science. 277:1508-1510.

5. Saville, B., et al. 2000. Ligand-, cell-, and estrogen receptor subtype (alpha/beta)-dependent activation at GC-rich (Sp1) promoter elements. J. Biol. Chem. 275:5379-5387.

6. Szego, C.M., and Davis, J.S. 1967. Adenosine 3',5' monophosphate in rat uterus: acute elevation by estrogen. Proc. Natl. Acad. Sci. U. S. A. 58:1711-1718.

7. Pietras, R.J., and Szego, C.M. 1977. Specific bind- ing sites for oestrogen at the outer surfaces of isolated endometrial cells. Nature. 265:69-72.

8. Filardo, E.J., Quinn, J.A., Bland, K.I., and Frackelton, A.R., Jr. 2000. Estrogen-induced activation of Erk-1 and Erk-2 requires the G protein-coupled receptor homolog, GPR30, and occurs via transactivation of the epidermal growth factor receptor through release of HB-EGF. Mol. Endocrinol. 14:1649-1660

9. Kousteni, S., et al. 2001. Nongenotropic, sex-non specific signaling through the estrogen or androgen receptors: dissociation from transcriptional activity. Cell. 104:719-730.

10. Jilka, R.L., et al. 1992. Increased osteoclast development after estrogen loss: mediation by interleukin-6. Science. 257:88-91.

11. Kousteni, S., et al. 2002. Reversal of bone loss in mice by nongenotropic signaling of sex steroids. Science. 298:843-846.

12. Kousteni, S., et al. 2003. Kinase-mediated regulation of common transcription factors accounts for the bone-protective effects of sex steroids. J. Clin. Invest. 111:1651-1664. doi:10.1172/JCI200317261.

13. Manabe, N., et al. 2001. Connection between B lymphocyte and osteoclast differentiation pathways. J. Immunol. 167:2625-2631.

14. Sato, T., Shibata, T., Ikeda, K., and Watanabe, K. 2001. Generation of bone-resorbing osteoclasts from B220+ cells: its role in accelerated osteoclastogenesis due to estrogen deficiency. J. Bone Miner. Res. 16:2215-2221.

15. Katavic, V., et al. 2003. The surface antigen CD45R identifies a population of estrogen-regulated murine marrow cells that contain osteoclast precursors. Bone. In press.

16. Miller, G. 2002. Divorcing estrogen's bright and dark sides. Science. 298:723-724.

\title{
What is the real role of antimicrobial polypeptides that can mediate several other inflammatory responses?
}

\section{Peter Elsbach}

Department of Medicine, New York University School of Medicine, New York, New York, USA

Antimicrobial peptides are effector molecules of innate immunity with microbicidal and pro- or anti-inflammatory activities. Their role is now widening following evidence that one such multifunctional peptide, LL-37, induces angiogenesis, a process essential for host defense, wound healing, and tissue repair (see related article beginning on page 1665).

J. Clin. Invest. 111:1643-1645 (2003). doi:10.1172/JCI200318761.

The last two decades have heralded impressive progress in the identification of a broad array of structurally and functionally diverse polypeptides

\footnotetext{
Address correspondence to: Peter Elsbach, Department of Medicine, New York University School of Medicine, 550 First Avenue, New York, New York 10016, USA. Phone: (212) 263-5633; Fax: (212) 263-3952; E-mail: elsbap01@mcrcr.med.nyu.edu. Conflict of interest: The author has declared that no conflict of interest exists.
}

implicated in many aspects of the host response to infection and other inflammatory stimuli.

Almost half a century ago, Hirsch described the antimicrobial properties of phagocytin, a crude protein fraction of polymorphonuclear leukocytes (1), thereby setting the stage for a growing interest in the biologic activities of protein components of inflammatory cells. The subsequent exploration of the functional capabilities of defined proteins and peptides in inflammation paralleled the evolving methodology of protein biochemistry and purification, and molecular biology. In this historical context, the initial focus remained on the antibacterial action of newly isolated proteins and peptides, reflecting an ongoing search for new antibiotics and the relative ease of conducting bioassays of bacterial viability $(2,3)$.

\section{Discovery of proteins and polypeptides with antibacterial properties}

Numerous proteins and polypeptides with antimicrobial activity in vitro have now been isolated and/or cloned from a broad range of both simple and complex organisms, including humans. Thus, endogenous-polypeptide antibiotics became prominent targets in the study of antimicrobial host defenses per se and also as potential pharmacologic agents (3). Whole families of such gene products have been identified in plants, insects, and other animals (4) in settings that are consistent with prominent roles in innate immunity. How well have these roles been defined so far? 
Recruitment of inflammatory cells

Promote ingestion of microbes

Stimulate production and release of

pro- and anti-inflammatory agents

Antimicrobial action

Repair
Figure 1

Functions of "antimicrobial" polypeptides in innate immunity.

The initial demonstration of antimicrobial activity of isolated polypeptides in vitro, often under very artificial laboratory conditions and against nonpathogenic strains of bacteria, did not establish whether such polypeptides played a prominent role in vivo. Moreover, since these polypeptides do not act alone and many share the apparent molecular determinants of antimicrobial activity, elucidation of their role in a complex mix in vivo has remained elusive.

\section{Polypeptides with antimicrobial and other biologic activities}

Uncertainty grew concerning the actions and effects of individual polypeptides after several were found to possess other biologic activities, apparently unrelated to their antimicrobial actions (Figure 1). For example, $\alpha$-defensins, at concentrations far lower than are required for their antimicrobial action, are capable of several other functions such as chemotactic and corticostatin-like actions (5).

In this issue of the JCI, Koczulla et al. (6) identify yet another novel biologic activity of the polypeptide LL-37/ hCAP-18, initially described as an antimicrobial member of the cathelicidin family (7). Cathelicidins, first isolated from polymorphonuclear leukocytes, are also constituents of lining epithelial cells (6). Proteolytic cleavage of cathelicidin proforms is required to free the potent antibacterial activity of the C-terminal peptide LL-37/hCAP-18. The uncleaved proform is devoid of antibacterial activity. However, at subnanomolar concentrations, it is capable of blocking the bioactivity of endotoxin (8). Koczulla et al. now show that $\mu \mathrm{g} / \mathrm{ml}$ concentrations of synthetic LL-37/
hCAP-18 stimulate angiogenesis both in vitro and in vivo (6). The proliferative effect on endothelial cells and vessel formation is convincingly demonstrated under tissue culture conditions. These effects are also manifest in vivo: administration of LL-37 was shown to stimulate capillary formation in a rabbit hindlimb model of ischemia, and mice deficient in the murine analog of LL-37/hCAP-18 demonstrated less neovascularization of skin lesions than wild-type controls. Both the chemoattractant and the angiogenic actions of LL-37 are thought to be receptor-mediated by interaction with formyl peptide receptor-like 1 , found on macrophages, neutrophils, and subsets of lymphocytes (6). It is particularly interesting that upon exposure of human umbilical cord endothelial cells to human serum (10\%), which was previously shown to inhibit the antimicrobial activity and anti-host cell cytotoxic action of LL-37 (6), the angiogenic activity of LL-37 was unchanged.

The angiogenic activity of exogenous LL-37, as demonstrated in the animal experiments, does not reveal whether endogenous LL-37 participates in the regulation of neovascularization by the many other angiogenic factors that operate in vivo. Also, what governs the release of the active peptide from its precursor during inflammation is largely unknown.

The recent report that the ribonuclease angiogenin, a component of specialized epithelial cells of the small intestinal crypt known as Paneth cells, is also microbicidal (9) further illustrates that such agents may be multifunctional. Obviously, it is a formidable task to define the (patho)physiologic contribution of any individual agent to in vivo events where multiple agonists and antagonists, at different concentrations and with different affinities, compete for the same targets.

Whatever the primary function of these peptides, the study by Koczulla et al. (6) adds to the growing evidence that polypeptides produced, stored, and secreted by inflammatory and epithelial lining cells may have more than one role in the complex innate immune responses of the host. This recognition raises new issues: much of the interest in the pursuit of these polypeptides is linked to the hope that synthetic or recombinant derivatives of bioactive polypeptides, such as LL-37/hCAP-18, may have a future as administered agents for the treatment of infection and its sequelae. However, if such agents participate in more than one biologic activity, possibly at different stages in an inflammatory process, the evaluation of their usefulness and safety becomes accordingly more elaborate. Is it reasonable, then, to anticipate the addition of such bioactive substances to the pharmacopoeia as treatments of complex clinical disorders? Notwithstanding the unknowns sketched here that require clarification, the answer is probably yes.

Recent reports suggest a role for a cathelicidin common to both mice and humans, in addition to other endogenous polypeptides with antibacterial activity, in providing protection against skin infections (10-12). Other administered proteins and peptides have been shown to protect animals against systemic infections $(3,13)$.

When disease and/or treatment deplete host stores of proteins and peptides with defense functions and impair or overwhelm their production by both myeloid and epithelial cells, replenishment with exogenous analogs may well become an important adjunctive therapy. This can be envisioned, for example, in cases of otherwise healthy children with severe pediatric bacterial infections that do not respond to available antibiotics $(14,15)$.

\footnotetext{
1. Hirsch, J.G. 1956. Phagocytin: a bactericidal substance from polymorphonuclear leukocytes. J. Exp. Med. 103:589-621.

2.Ganz, T., and Weiss, J. 1997. Antimicrobial peptides of phagocytes and epithelia. Semin. Hematol. 34:343-354.

3. Elsbach, P., Weiss, J., and Levy, O. 1999. Oxygen-
} 
independent antimicrobial systems of phagocytes. In Inflammation. Basic principles and clinical correlates. 3rd edition. J. Gallin and R. Snyderman, editors. Lippincott-Raven. Philadelphia, Pennsylvania, USA. 801-817.

4. Hoffmann, J.A., Kafatos, F.C., Janeway, C.A., and Ezekowitz, R.A. 1999. Phylogenetic perspectives in innate immunity. Science. 284:1313-1318.

5. Bateman, A., Singh, A., Shustik, C., Mars, W.M., and Solomon, S. 1991. The isolation and identification of multiple forms of the neutrophil granule peptides from human leukemic cells. J. Biol. Chem. 266:7524-7530.

6. Koczulla, R., et al. 2003. An angiogenic role for the human peptide antibiotic LL-37/hCAP-18. J. Clin. Invest. 111:1665-1672. doi:10.1172/JCI200317545.
7.Zanetti, M., Gennaro, R., Scocchi, M., and Skerlavaj, B. 2000. Structure and biology of cathelicidins. Adv. Exp. Med. Biol. 479:203-218.

8. Zarember, K.A., et al. 2002. Host defense functions of proteolytically processed and parent (unprocessed) cathelicidins of rabbit granulocytes. Infect. Immun. 70:569-576.

9. Ganz, T. 2003. Angiogenin: an antimicrobial ribonuclease. Nat. Immunol. 4:213-214.

10. Ong, P.Y., et al. 2002. Endogenous antimicrobial peptides and skin infections in atopic dermatitis. N. Engl. J. Med. 347:1151-1160.

11. Cole, A.M., et al. 2001. Inhibition of neutrophil elastase prevents cathelicidin activation and impairs clearance of bacteria from wounds. Blood. 97:297-304
12. Nizet, V., et al. 2001. Innate antimicrobial peptide protects the skin from invasive bacterial infection. Nature. 414:454-457.

13. Levy, O. 2002. Therapeutic potential of the bactericidal/permeability-increasing protein. Expert Opin. Investig. Drugs. 11:159-167.

14. Levy, O., et al. 1999. Impaired innate immunity in the newborn: newborn neutrophils are deficient in bactericidal/permeability-increasing protein. Pediatrics. 104:1327-1333.

15. Levin, M., et al. 2000. Recombinant bacterici$\mathrm{dal} /$ permeability-increasing protein (rBPI21) as adjunctive treatment for children with severe meningococcal sepsis: a randomised trial. rBPI21 Meningococcal Sepsis Study Group. Lancet 356:961-967.

\title{
Functionally mature virus-specific $\mathrm{CD8}^{+}$ $T$ memory cells in congenitally infected newborns: proof of principle for neonatal vaccination?
}

\author{
P.G. Holt
}

Telethon Institute for Child Health Research and Centre for Child Health Research, Faculty of Medicine and Dentistry, University of Western Australia, Perth, Western Australia, Australia

The presence in newborns of a mature and functional $\mathrm{CD}^{+} \mathrm{T}$ cell response to congenital cytomegalovirus infection (see related article beginning on page 1747) suggests that the machinery necessary to prime such responses is present in utero and raises questions related to neonatal vaccination.

J. Clin. Invest. 111:1645-1647 (2003). doi:10.1172/JCI200318805.

In this issue of the JCI, Marchant et al. (1) report the presence of functionally mature cytolytic CD8 ${ }^{+} \mathrm{T}$ lymphocytes in newborns with congenital cytomegalovirus (HCMV) infection. This finding adds to the growing body of evidence suggesting that intrauterine antigenic stimulation has the potential to elicit protective immunity in the fetus, which persists into the newborn period. This has important implications in relation to infant vaccine development, but significant questions remain to be answered before this potential can be fruitfully exploited.

Address correspondence to: P. G. Holt, Division of Cell Biology, Telethon Institute for Child Health Research, PO Box 855, West Perth, Western Australia 6872, Australia. Phone: 61-8-9489-7838; Fax: 61-8-9489-7707; E-mail: patrick@ichr.uwa.edu.au.

Conflict of interest: The author has declared that no conflict of interest exists.

Nonstandard abbreviations used: human cytomegalovirus (HCMV); respiratory syncytial virus (RSV).

\section{Susceptibility to infectious disease during infancy}

Increased susceptibility to infectious diseases is an inescapable fact of neonatal life and is generally ascribed to developmentally related deficiencies in immune function, which are incompletely understood. Recent advances in developmental immunology have provided fresh insight into this issue. Of particular relevance are studies investigating the mechanisms that facilitate fetal survival in the face of continuous maternal immune surveillance. This body of research (reviewed in ref. 2) has demonstrated that Th1-type cytokines (especially IFN- $\gamma$ ) are exquisitely toxic to the placenta, and their release at the fetomaternal interface, triggered by antiallograft or antimicrobial responses, is an important cause of premature termination of pregnancy $(2,3)$.

In the face of these challenges to fetal survival, evolution has fashioned a range of overlapping control mechanisms to regulate expression of immunity at the fetomaternal interface. The most potent of these involve local placental production of tryptophan metabolites (4) and IL-10 (5), which inhibit $\mathrm{T}$ cell activation and proliferation, and expression of FasL, which eliminates activated T cells (6). A second tier of immunomodulatory mechanisms selectively dampens Th1 immunity via local production of a range of molecules that are Th2 trophic and/or Th1 inhibitory. These include production by placental trophoblasts of IL-10 and prostaglandin E2, which drive Th2 switching $(5,7)$, and progesterone, which inhibits IFN- $\gamma$ gene transcription (8). Collectively, these mechanisms attenuate fetal capacity to develop active immunity and bias any responses that escape immunosuppression toward the Th2 phenotype.

Survival in the microbiologically hostile postnatal environment necessitates upregulation of immune (in particular Th1) functions, which is mediated via microbial pattern recognition receptors (9). However, the postnatal maturation of Th 1 competence proceeds relatively slowly between birth and weaning, and this appears to underlie the generally attenuated capacity of infants to efficiently generate $\mathrm{CD}^{+} \mathrm{T}$ cell memory to antigens in or on pathogens and in response to vaccines $(10,11)$. This may be attributable in part to deficient production of IL-12 (12), which is required for stabilization of the IFN- $\gamma$ transcriptional machinery in T cells (13), and also to hypermethylation of the promoter of the IFN- $\gamma$ gene in neonatal $\mathrm{CD}^{+} \mathrm{T}$ cells, which directly inhibits transcription (14). Moreover, recent studies indicate that $\geq 90 \%$ of circulating $\mathrm{CD}^{+}$ $\mathrm{CD}_{45 \mathrm{RO}^{-}}$naive $\mathrm{T}$ cells in neonates are 